\title{
Low grade astrocytoma transformating primitive neuroectodermal tumour in an adult? A case report and review of literature
}

Xiaofeng Wang ${ }^{1,2,3}$, Yuqiang Sun ${ }^{2}$, Zeshi Tan ${ }^{2}$, Anlong $\mathrm{Ji}^{4}$, Xu Sun ${ }^{2}$, Xinyu $\mathrm{Li}^{2}$, Ningwei Che ${ }^{2}$, Kang Yang ${ }^{2}$, Yaoling Liü ${ }^{5}$ Xiaodong Luo ${ }^{2}$, Guanyu Wang ${ }^{2}$, Lan Luan², Minghai Wei ${ }^{2^{*}}$ and Jian Yin ${ }^{2,3^{*}}$

\begin{abstract}
Background: Supratentorial primitive neuroectodermal tumors (sPNET) are rarely occurred in adults. Only 39 cases have been reported in the past decade. The transformation from low grade to aggressive astrocytoma is well known. However, such convert from a low-grade astrocytoma (LGA) into a sPNET between two completely different tumors is rarer.

Case Presentation: This report discussed a 36-year-old male, who presented with the chief complaint of partial seizures and left limbs dysfunction and Magnetic resonance imaging (MRI) of whom revealed right frontal lesions which was operated to resect accompanied by the histological diagnosis of SPNET. The patient underwent operation 2.5 years ago because of seizures and was diagnosed with right frontal astrocytoma confirmed by pathology. However, radiotherapy was not preformed on him after the primary surgery. Histology now revealed high grade PNET.

Conclusion: It is exactly uncertain that reports revelant to transformation from low-grade astrocytoma to a new kind of tumor or neoplasm induced by radiotation have been published. This case report is accompanied by a review of 39 cases of adult SPNET in the past decade, especially discussing about the transformation of low grade glioma into sPNET.
\end{abstract}

Keywords: SPNET, LGA, Adult

\section{Background}

Primitive neuroectodermal tumor of the central nervous system (CNS) described by Hart and Earle in 1973 [1] is embryonal tumors composed of undifferentiated neuroepithelial cells which are capable of both glial and neuronal differentiation. The definition based on the morphology is focused on small, blue round cells with a high nuclear-cytoplasmic ratio exhibiting frequent mitosis, apoptosis and neuronal marker positivity. Supratentorial primitive neuroectodermal tumor (sPNET) is defined as a cerebrum or suprasellar embryonal tumor made up of undifferentiated or poorly differentiated neuroepithelial cells which have the capacity for displaying divergent differentiation along neuronal, astrocytic,

\footnotetext{
* Correspondence: weimingh@sina.cn; dyeyDr_yin@163.com

${ }^{2}$ Department of Neurosurgery, The Second Affiliated Hospital of Dalian

Medical University, 467 Zhongshan Road, Shahekou, Dalian, Liaoning 116023,

People's Republic of China

Full list of author information is available at the end of the article
}

ependymal, muscular or melanocytic lines according to the World health Organization (WHO) [2]. Although PNET accounts for $2.8 \%$ of all primary cerebral tumors of childhood and adolescence [3], PNETs are extremely rare in adults, representing less than $0.5 \%$ of all intracranial tumors [4]. So far only 39 such cases have been previously reported in the past decade.

\section{Case presentation}

A 36-year-old male who had a 2-month history of tonicclonic seizures and 2.5-year history of headache consulted an Epilepsy center. According to his medical history, a right frontal lobe lesion was resected 2.5 years ago in another hospital, and the pathologically diagnose was diffuse astrocytoma (WHO II) (Fig. 2a) while the adjuvant radio-chemotherapy was not performed.

Although oxcarbazepine had been taken in to control epilepsy, he still presented with increased seizure frequency, alongside with gradual dizziness and liability to 
emotional irritation for 2 months. Focal neurological deficit of left limbs and muscle weakness had been detected through physical examination. The MRI revealed recurrence of tumor located in right frontal lobe, which was iso-low-intense on T1 weighted images, highintense on T2 weighted images, but MRI also showed a mixed-anomaly signal in both T1WI and T2WI, which was composed of cyst fluid surrounded by brain tissues (Fig. 1a, b). It demonstrates enhancement within tumor, which appears enlarged (Fig. 1c). During the craniotomy, the dura matter was found to be normal, while the surface of the brain was found to be deep yellow. Along the tissue of cerebral surface resection, the old cystic fluid was found and removed. The tumor was subtotal resected.

Histologically, this tumor was confirmed to be a typical diffuse astrocytoma (Fig. 2a) after the first surgery, which was in a prominent microcystic background from the H\&E stain. The recurrent tumor was hypercellular with small round cells containing hyperchromatic nuclei and a high nucleo-cytoplasmic ratio (Fig. 2b). There were many cells with irregular nuclei. The tumor cells were negative for argyrophil fibers stain, but the Homer-wright (Fig. 2b, c) can be detected distinctly. According to an immunohistochemical analysis, the tumor was reactive to CD99 (Fig. 2d), glial fibrillary acidic protein (GFAP) (Fig. 2e), synaptophysin (Fig. 2f), neuron specific enolase (Fig. 2g), Vimentin (Fig. 2h), CD56 (N-CAM) (Fig. 2i), The tumor was thus diagnosed to be sPNET [5] with glial, mesenchyimal, neuronal differentiation. The Ki67 index was about $17 \%$. The tumor was negative for Oligo-2, AE1/AE3 and S-100. Based on these findings, this tumor was diagnosed to be supratentorial central PNET. The patient did not get any radiotherapy or chemotherapy [6] on account of the low grade astrocytoma. But the recurrent tumor was PNET (WHO IV) [7]. Radiographic follow-up for 5 month showed no evidence of recurrence.

\section{Discussion}

PNETs comprise a heterogeneous group of malignant tumors that mainly affect the pediatric population, which are composed of neuroepithelial cells that are able to differentiate along the neuronal, astrocytic, muscular, or melanocytic lines, with high index of proliferation [8]. Supratentorial PNETs are very rare and aggressive tumors (WHO IV), and account for approximately 2.5$3 \%$ of all pediatric tumors [9]. LGA in adults are primary (CNS) neoplasms with a variable biological behaviour. While some remain dormant for several years, others grow slowly, infiltrating surrounding normal brain and showing an intrinsic tendency for transformation into a higher grade astrocytoma [10].

Generally, adult PNETs usually appear as lobulated, purple-grayish or pinkish masses. On microscopic examination, the tumor cell population consists of undifferentiated small cells with ill-defined, scanty cytoplasm, and round or oval cells with hyperchromatic nuclei [11]. Mitotic activity is variable. Microscopic calcifications, necroses and Homer-Wright rosettes are also observed in a number of cases. This article summarize a total of 40 cases of PNET in the past decade, most pathological diagnosis is confirmed by tumor cell morphology. Pathology revealed SPNET that was characterized by dense cellularity, marked nuclear pleomorphism, nuclear molding, brisk mitotic activity, and abundant apoptotic cell death. The sPNET is diagnosed by Homer-Wright rosettes of morphological and immunohistochemical positive expression such as CD99 and GFAP, Syn, CD56 which alleviate the diagnostic rate. The Ki-67 index is an important basis of pathological diagnosis.

Nowdays, the genetic and biological features of pediatric PNETs are becoming more and more popular. There are several theories that have been postulated to describe the pathogenesis of radiogenic tumors: (a) radiation could cause genetic alterations by disrupting the deoxyribonucleic acid (DNA) of specific genes, such as PTEN or the p53 tumor suppressor gene [12, 13]; (b)

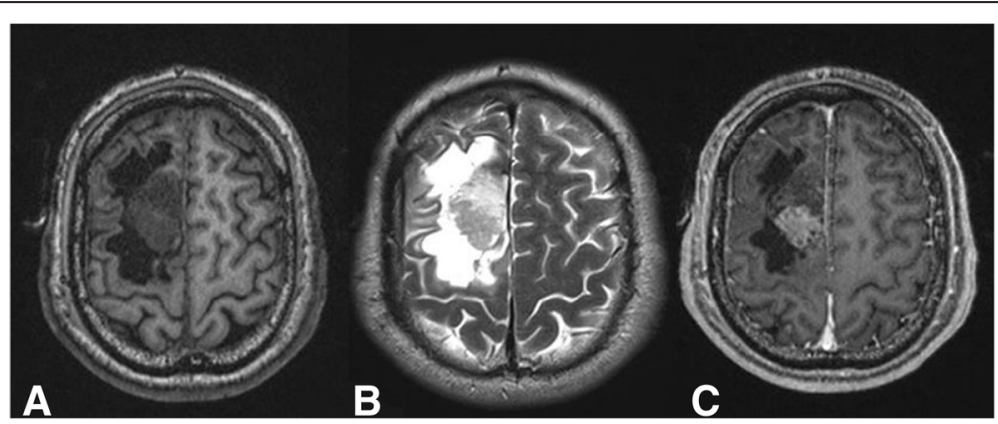

Fig. $1 \mathrm{MRI}$ scan with contrast: a T1WI, b T2WI, c enhancement: iso-low- intense on T1 weighted images, high- intense on T2 weighted images, but MRI also showed a mixed-anomaly signal in both T1WI and T2WI, which was composed of cyst fluid surrounded by brain tissues, enhancement within tumour, which appears enlarged 


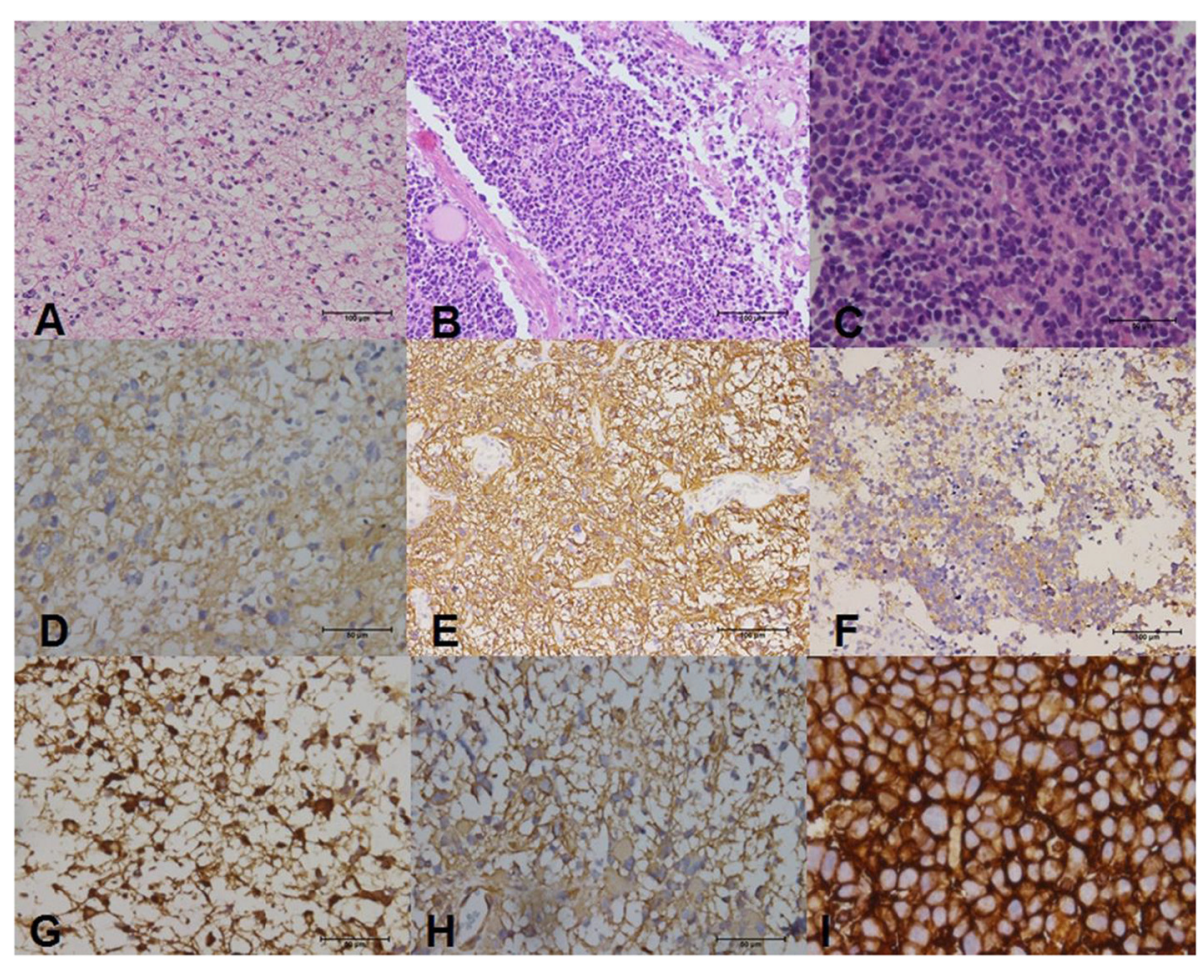

Fig. 2 Histopathologic and immunohistochemical features in this case of adult PNETs.: a a diffuse astrocytoma: a prominent microcystic background from the H\&E stain. $\mathbf{b}$ Homer-Wright rosettes. c Tumors were mainly composed of undifferentiated tumor cells, with hyperchromatic nuclei. d CD99 (+). e GFAP(+). f synaptophysin(+). g NSE(+). h Vimentin(+). i CD56(N-CAM) (+)

karyotypic instability results from the radiation-induced multiple chromosome aberrations [14]; (c) irradiation may induce the release of oncogenic factors, such as platelet-derived and vascular endothelial growth factors [15], beta chain of fibroblast growth factor [16], and raised tissue level of basic fibroblast growth factor [17] by alterating vascular parenchymal. In a patient with radio-induced glioma reported by Gessi et al. [18], the genetic alterations were p53 mutation, in which the loss of heterozygosity of $17 \mathrm{p}$ and $19 \mathrm{q}$, and O6methylguanine-DNA methyl-transferase (MGMT) promoter methylation occurred, and without amplification of epidermal growth factor receptor (EGFR). An unusual somatic p53 mutation of a type specific to radiationinduced DNA damage in a secondary glioblastoma identified by Tada [12]. Also IL-1ßm-RNA was seven-fold higher in the recurrent tumor of PNET and it could have triggered the fatal vasospasm [19]. Signaling pathways, sonic hedgehog (Shh), Wnt, and Notch pathways, especially the Shh pathway, are considered playing an important role in the pathogenesis and biological behavior of desmoplastic PNET [20]. Recent studies indicated inappropriate activation of Shh pathway promotes tumorigenesis and increases the risk of tumor development by ionizing radiation [21]. Mutations in genes in the Shh pathway appear to contribute to PNET development.
This case is extremely rare in the same parts of the two different tumors, PNET and LGA. Clinical features as well as pathological and molecular studies can discriminate PNET from astrocytoma, as these tumors are clearly different in terms of their origin, typical features and common characteristics. The pathogenesis of secondary sPNET is unknown. Whether they represent transformation of LGA, radiotherapy induced or de novo tumor is not clear. The possible explanations for occurrence of multiple diverse primary brain tumors in cases that contain two different tumors include secondary brain tumor induction by radiation, tumorigenetic activities of certain oncogenes, and defects in tumor suppressor genes [22]. According to the modified Cahan's criteria for radiation-induced tumors: the onset of secondary tumors is sited in the previously irradiated field, both the primary and secondary tumors are histologically different from each other, and a sufficiently long latency period [23]. There were two cases tranlated from low grade gliomas to PNET with pathologic supports, which might be related to radiotherapy directly, but still not enough [24]. D. PAL analyzed 12 cases which were secondary pathological certification in PNET patients retrospectivly, including 10 cases directly related to radiation therapy, and 2 cases of a direct tumor level change [17]. The pathological type of this case is also a direct 
shift, but it still doesn't have enough reasons to be prove the origin. Regardless, the strategy of treatment was still following the current one. After the secondary surgery, we performed radiotherapy focused on the tumor position. Now 5 months later, we did not see enough evidence of recurrence.

sPNETs are considered as a challenging clinical entity for neurosurgeons due to its poor prognosis it confers. Intrinsic biological difference of these tumors has been suggested by sPNETs patients' worse outcome in terms of survival rates in comparison with high risk medulloblastoma patients treated by the same therapeutic strategy [9]. The overall 5-year progression-free survival rate for patients who do not undergo resection ranges from 18 to $47 \%$ [9]. The reported overall survival of PNETs in the pediatric population varied between 29 and $57 \%$ [11]. Follow-up ranged from $48 \mathrm{~h}$ to 204 months (mean 22.7 months; median 18.7 months) for this summary. 25 patients $(62.5 \%)$ were dead, 15 patients were alive. For all patients, the estimated OS rates were $63.6 \%$ at 1 year, $21.2 \%$ at 2 years, $12.1 \%$ at 3 years, and $6.1 \%$ at 5 years (Fig. 3, Additional file 1: Table S1). The mean duration of overall survival (OS) was 22 months. The survival time differences could be probably attributed to differences of the tumor sites, growth ways, proliferation differentiation degrees and the specific treatments. Maybe a larger sample-controlled trials is needed. Moreover, the prognosis for adults with supratentorial PNETs seems to be worse than that of their pediatric counterparts [25]. In adults the most important prognostic factor is considered to be the Ki-67 index. Kim et al. [4] reported that adult patients with a Ki-67 index greater than $30 \%$ demonstrated very poor outcome, with a mean postoperative survival time of eight months [26]. Mutations in IDH1 were identified in $6 \%$ primary sPNET [27]. More importantly, in a study by Hayden et al., it has been suggested that mutations in IDH1, which encodes for cytoplasmic isocitrate dehydrogenase, are among the most frequent mutations in sPNET in adult cases [28]. This case is that no radiotherapy one was administrated but transforming from another tumor.

It is not clear whether the occurrence of sPNET in our patient represent transformation or de novo tumors. Given the similarity to few previous cases, we postulate that they are probably radiation induced which should be considered in differential diagnosis. Molecular abnormalities, oncogenes or defective tumor-suppressor genes of medulloblastomas and astrocytomas patients have recently been investigated; however, corresponding molecular data of the present patients are unknown. The potential factors responsible for the development of multiple primary brain tumors remain unknown, further studies in elucidating the relevant factors are wanted. In addition, the more complex multifactors inducing tumorigenesis have to be clarified in order to resolve the mechanisms of multiple primary brain tumors. Future studies in large series may provide reliable data on the origin and prognostic significance of this component.

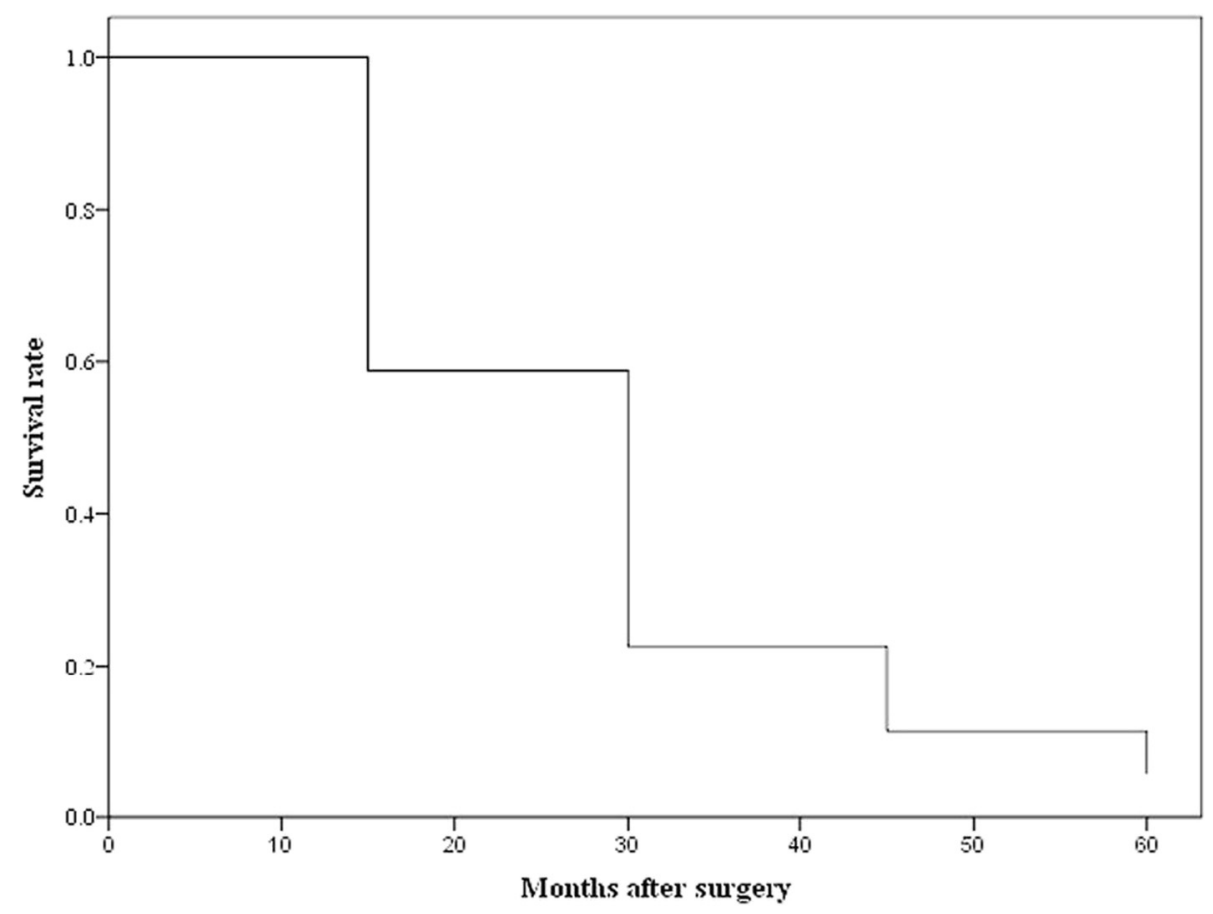

Fig. 3 Survival time for 40 sPNET adults in the past decade 


\section{Conclusion}

This case is exactly uncertain that reports revelant to transformation from low-grade astrocytoma to a new kind of tumor or neoplasm induced by radiotation have been published. It is not clear whether the occurrence of sPNET in our patient represent transformation or de novo tumors, However, radiotherapy was not preformed on the patient after the primary surgery. This paper is accompanied by a review of 40 cases of adult sPNET in the past decade, for all patients, the estimated OS rates were $63.6 \%$ at 1 year, $21.2 \%$ at 2 years, $12.1 \%$ at 3 years, and $6.1 \%$ at 5 years.

\section{Additional file}

Additional file 1: Table S1. Summary of clinical and histological

features of cases in the past decade. (DOC $151 \mathrm{~kb}$ )

\section{Abbreviations}

CNS, Central nervous system; LGA, low-grade astrocytoma; MRI, magnetic resonance image; OS, overall survival; sPNET, Supratentorial primitive neuroectodermal tumor

\section{Acknowledgements}

The authors have no personal financial or institutional interest in any of the drugs, materials, or devices described in this article. We thank the physicians who referred the patients to surgery.

\section{Authors' contributions}

All authors read and approved the final manuscript.

\section{Competing interests}

The authors declare that they have no competing interests.

\section{Ethics approval and consent to participate}

We obtained written permission from the patient to publish this case report. This study was approved by the ethics committee of The Second Affiliated Hospital of Dalian Medical University.

\section{Author details \\ 'Department of Neurosurgery, Weinan Central Hospital, Weinan, Shaanxi 714000, People's Republic of China. ${ }^{2}$ Department of Neurosurgery, The Second Affiliated Hospital of Dalian Medical University, 467 Zhongshan Road, Shahekou, Dalian, Liaoning 116023, People's Republic of China. ${ }^{3}$ Epileptic Center of Liaoning, The Second Affiliated Hospital of Dalian Medical University, Dalian, Liaoning 116023, People's Republic of China. ${ }^{4}$ Department of General Surgery, The Second Affiliated Hospital of Dalian Medical University, Dalian, Liaoning 116023, People's Republic of China. ${ }^{5}$ Department of Neurosurgery, Affiliated Fuxing Hospital, The Capital University of Medical Sciences, Beijing 100038, China.}

Received: 19 February 2016 Accepted: 3 June 2016

Published online: 18 July 2016

\section{References}

1. Hart MN, Earle KM. Primitive neuroectodermal tumors of the brain in children. Cancer. 1973:32(4):890-7.

2. Rorke LB, Hart MN, Mclendon RE. Supratentorial primitive neurorctodermal tumor (PNET). In: Kleihues P, Cavenee WK, editors. Pathology \& Genetics: tumors of the nervous system. Lyon: LARC Press; 2000. p. 141-4.

3. Gaffney CC, Sloane JP, Bradley NJ, Bloom HJ. Primitive neuroectodermal tumours of the cerebrum, pathology and treatment. J Neurooncol. 1985; 3(1):23-33.

4. Kim DG, Lee DY, Paek SH, Chi JG, Choe G, Jung HW. Supratentorial primitive neuroectodermal tumors in adults. J Neurooncol. 2002;60(1):43-52.

5. Becker LE, Giangaspero F, Rorke LB, et al. Embryonal tumours. In: Kleihues P, Cavenee WK, editors. World health organization classification of tumours, pathology and genetics of tumours of the nervous system. Lyon: IARC Press; 2000. p. 123-48.

6. Cohen BH, Zeltzer PM, Boyett JM, et al. Prognostic factors and treatment results for supratentorial primitive neuroectodermal tumors in children using radiation and chemotherapy: a Children's Cancer Group randomized trial. J Clin Oncol. 1995;13(7):1687-96.

7. McLendon RE, Judkins AR, Eberhart CG, et al. Central nervous system primitive neuroectodermal tumours. In: Louis DN, Ohgaki H, Wiestler OD, et al, eds. WHO Classification of Tumours of the Central Nervous System. Lyon: IARC press; 2007, 141-146

8. Louis DN, Ohgaki H, Wiestler OD, Cavenee WK, Burger PC, Jouvet A, et al. The 2007 WHO classification of tumours of the central nervous system. Acta Neuropathol. 2007;114:97-109.

9. Jakacki RI. Pineal and nonpineal supratentorial primitive neuroectodermal tumors. Childs Nerv Syst. 1999:15:586-91.

10. Wessels PH, Weber EJW, Raven G, et al. Supratentorial grade II astrocytoma: biological features and clinical course. Lancet Neurol. 2003;2:395-403.

11. Behdad A, Perry A. Central nervous system primitive neuroectodermal tumors: A clinicopathologic and genetic study of 33 cases. Brain Pathol. 2010;20:441-50.

12. Tada M, Sawamura $Y$, Abe $H$, Iggo R. Homozygous p53 gene mutation in a radiation-induced glioblastoma 10 years after treatment for an intracranial germ cell tumor: case report. Neurosurgery. 1997:40(2):393-6.

13. Chang SM, Barker 2nd FG, Larson DA, Bollen AW, Prados MD. Sarcomas subsequent to cranial irradiation. Neurosurgery. 1995:36(4):685-90.

14. Sachs RK, Hlatky LR, Trask BJ. Radiation-produced chromosome aberrations: colourful clues. Trends Genet. 2000;16(4):143-6.

15. Li $M$, Jendrossek $V$, Belka $C$. The role of PDGF in radiation oncology. Radiat Oncol. 2007:11:2-5.

16. Liwnicz BH, Berger TS, Liwnicz RG, Aron BS. Radiation-associated gliomas: a report of four cases and analysis of postradiation tumors of the central nervous system. Neurosurgery. 1985;17(3):436-45.

17. Rothbart D, Awad IA, Lee J, Kim J, Harbaugh R, Criscuolo GR. Expression of angiogenic factors and structural proteins in central nervous system vascular malformations. Neurosurgery. 1996;38(5):915-24. discussion 924-5.

18. Gessi M, Maderna E, Guzzetti S, Cefalo G, Massimino M, Solero CL, et al. Radiation-induced glioblastoma in a medulloblastoma patient: a case report with molecular features. Neuropathology. 2008:28(6):633-9.

19. Miscusi M, Martino LD, Antonelli M, Mangino G, Ricciardi L, Spinelli G, et al. A case of fatal late vasospasm in a patient with a recurrent, supratentorial rhabdoid primitive neuroectodermal tumor: possible molecular implications. Turk Neurosurg. 2014;24(5):793-8.

20. de Bont JM, Packer RJ, Michiels EM, den Boer ML, Pieters R. Biological background of pediatric medulloblastoma and ependymoma: a review from a translational research perspective. Neuro Oncol. 2008;10(6):1040-60.

21. Leonard JM, Ye H, Wetmore C, Karnitz LM. Sonic Hedgehog signaling impairs ionizing radiation-induced checkpoint activation and induces genomic instability. J Cell Biol. 2008;183(3):385-91.

22. Takeshi N, Kazuhiko S, Koji I, Toshikazu H, Kaoru K. A case of adult onset medulloblastoma during maintenance chemotherapy for anaplastic astrocytoma one year after radiotherapy. Turk Neurosurg. 2013;23(4):521-6.

23. Cahan WG, Woodard HQ, Higinbotham NL, Stewart FW, Coley BL. Sarcoma arising in irradiated bone; report of 11 cases. 1948. Cancer. 1998:82(1):8-34.

24. Chan MD, Attia A, Tatter SB, Lesser G, Zapadka ME, Mott RT, et al. Radiationinduced adult medulloblastoma: a two-case report and review of the literature. J Neurooncol. 2011:103(3):745-9.

25. Visée S, Soltner C, Rialland X, Machet MC, Loussouarn D, Milinkevitch S, et al Supratentorial primitive neuroectodermal tumours of the brain: multidirectional differentiation does not influence prognosis. A clinicopathological report of 18 patients. Histopathology. 2005:46(4):403-12.

26. Abuzayed B, Khreisat W, Maaiah W, Agailat S. Supratentorial primitive neuroectodermal tumor presenting with intracranial hemorrhage in adult. J Neurosci Rural Pract. 2014;5(2):176-9.

27. Hayden JT, Frühwald MC, Hasselblatt M, Ellison DW, Bailey S, Clifford SC. Frequent IDH1 mutations in supratentorial primitive neuroectodermal tumors (sPNET) of adults but not children. Cell Cycle. 2009:8(11):1806-7.

28. Lawandy S, Hariri OR, Miulli DE, Amin J, Minasian T, Gupta RK, et al. Supratentorial primitive neuroectodermal tumor in an adult: a case report and review of the literature. J Med Case Rep. 2012;6:361. 